\title{
QUANTIFICATION OF THE OCULAR REACTIONS TO MICROFILARICIDES IN THE CHEMOTHERAPY OF ONCHOCERCIASIS
}

\author{
M. HERO $\dagger$, A. C. BIRD*, K. AWADZI $\dagger$ \\ Ghana and London
}

\begin{abstract}
SUMMARY
Severe adverse systemic and ocular reactions have complicated the chemotherapy of onchocerciasis. A method for the quantification of ocular reactions to chemotherapy has been devised at the Onchocerciasis Chemotherapy Research Centre, at Hohoe, in Ghana. Symptoms, visual function, anterior segment inflammation and disease of the posterior segment are graded. The information is entered into a computerised database which allows reaction scores to be calculated both for individual patients, and for treatment groups. This system enables comparision of adverse ocular reactions to various new chemotherapeutic regimens.
\end{abstract}

Onchoceriasis, a parasitic disease caused by the nematode worm Onchocerca volvulus, is a major cause of preventable blindness in Africa, Central and South America. Chemotherapy of onchocerciasis has been complicated by severe systemic ${ }^{1-3}$ and ocular-6 reactions induced by death of microfilariae. Severe symptoms following drug administration may lead to non-compliance with therapy, and ocular inflammation may precipitate or aggravate visual disability. Methods to quantify systemic reactions during therapy have been devised, ${ }^{7-9}$ and recently an ocular reaction index based only on anterior segment inflammatory signs and ocular microfilarial loads was described. ${ }^{10}$ The posterior segment changes were not addressed although it is reactions in the retina and optic nerve which represent the major risk of therapy. For this reason a new assessment method has been created which provides a more complete index of ocular disease. The method described in this paper includes symptoms, changes in visual function, anterior segment inflammation and disease of the posterior segment. This complements the systemic assessment which is carried out routinely at the Onchocerciasis Chemotherapy Research Centre (OCRC).

†From Onchocerciasis Chemotherapy Research Centre, Hohoe Hospital, PO Box 144, Hohoe, Ghana.

* Department of Clinical Ophthalmology, Institute of Ophthalmology, Moorfields Eye Hospital, City Road, London EC1V 2PD.

Correspondence to Professor A. C. Bird, Moorfields Eye Hospital, City Road, London EC IN 2PD.

\section{METHODS}

Ocular symptoms: A detailed history is taken and patients are specifically asked about the presence of ocular itching, visual loss, photophobia and lacrimation. A score of five points is awarded if symptoms are present.

Visual function: Uncorrected visual acuity with each eye is measured at six metres using an illiterate ' $E$ ' chart, and scored. (Table I)

Visual fields are recorded using a standardised Goldmann perimeter with $\mathrm{I} / 4 \mathrm{e}$ and $\mathrm{I} / 2 \mathrm{e}$ white targets. The $\mathrm{I} / 4 \mathrm{e}$ isopter is recorded from non-seeing to seeing areas to obtain a peripheral field. A similar field is recorded to $\mathrm{I} / 2 \mathrm{e}$. If loss to the $\mathrm{I} / 4 \mathrm{e}$ target is detected, a visual field is undertaken to the IV/4e. Intersects between the radial and circumferential lines on the Goldmann chart outside the seeing field within the central $50^{\circ}$ to IV $/ 4 \mathrm{e}$, within the central $40^{\circ}$ to $\mathrm{I} / 4 \mathrm{e}$ and within the central $20^{\circ} \mathrm{C}$ to the $\mathrm{I} / 2 \mathrm{e}$ targes are counted and scored. (Table II).

In addition static perimetry with targets presented randomly for at least one second is recorded with $\mathrm{I} / 2$ at eccentricities of $5^{\circ}, 10^{\circ}, 15^{\circ}$ and $20^{\circ}$ on alternate $30^{\circ}$ radial lines of the Goldmann chart plus the four quadrants within $5^{\circ}$ making a total of 52 presentations. Missed I/2e static presentations are also counted and scored, two missed presentations being allowed for the blind spot. (Table II) Scoring is therefore weighted to the detection of early field loss.

Anterior segment inflammation: Microfilarial death, whether spontaneous or drug induced, may result in a conjunctivitis, limbitis and onchocercal punctate keratitis. Anterior uveitis may also complicate the clinical picture. Although these changes do not usually result in blindness, they have been included as they represent host response to the parasite. During examination of the anterior segment, ocular parasites in the anterior chamber and cornea are counted, although these counts are not considered in the scoring of ocular reactions. Signs of inflammation are graded and scored. (Table III) Aqueous flare is quantified 
Table I. Scoring for visual acuity

\begin{tabular}{lccccccccccc}
\hline & & & & & & & & \multicolumn{3}{c}{$<6 / 60$} \\
VA & $6 / 4$ & $6 / 5$ & $6 / 6$ & $6 / 9$ & $6 / 12$ & $6 / 18$ & $6 / 24$ & $6 / 36$ & $6 / 60$ & but PL & NPL \\
\hline Score & 0 & 5 & 10 & 15 & 20 & 25 & 30 & 35 & 40 & 45 & 50 \\
\hline
\end{tabular}

Table II. Scoring system for visual fields

\begin{tabular}{lllll}
\hline Stimulus & \multicolumn{4}{c}{ Missed points } \\
\hline IV/4e kinetic & $1-12$ & $13-24$ & $25-48$ & $49-72$ \\
I/4e kinetic & $1-12$ & $13-24$ & $25-36$ & $36-60$ \\
1/2e kinetic & $1-12$ & $13-20$ & $21-28$ & $29-36$ \\
I/2e static & $3-12$ & $13-20$ & $21-35$ & $36-52$ \\
Score & 5 & 10 & 15 & 20 \\
\hline
\end{tabular}

Table III. Scoring system for anterior segment inflammation

\begin{tabular}{lcccc}
\hline Feature & \multicolumn{4}{c}{ Grade } \\
\hline $\begin{array}{l}\text { Conjunctivitis } \\
\text { Limbitis }\end{array}$ & $\begin{array}{c}\text { hyperaemia } \\
\text { dilated } \\
\text { capillaries }\end{array}$ & $\begin{array}{c}\text { Chemosis } \\
\text { limbal } \\
\text { oedema }\end{array}$ & & \\
Globular infiltrates & $1-5$ & $6-10$ & $11-20$ & $21+$ \\
$\begin{array}{l}\text { Punctate opacities } \\
\text { Anterior uveitis }\end{array}$ & $1-5$ & $6-10$ & $11-2$ & $21+$ \\
*cells/field & $1-10$ & $11-20$ & $21-40$ & $41+$ \\
$* *$ flare & seen with & seen with & seen with & plasmoid \\
& no filter & filter 1 & filter 2 & aqueous \\
Score & 5 & 10 & 15 & 20 \\
\hline
\end{tabular}

*Light beam at 45 degrees; slit $2 \mathrm{~mm}$ high by $0.5 \mathrm{~mm}$ wide. ${ }^{* *}$ Light beam at 45 degrees; slit $2 \mathrm{~mm}$ high by $0.1 \mathrm{~mm}$ wide.

(Filters 1 and 2 are neutral density filters built into the optical column of the slit lamp, filter 2 being the denser one).

by interposing the neutral density filters in the Haag-Streit optical column and noting when it becomes just visible in a beam of light $0.2 \mathrm{~mm}$ wide at an angle of $45^{\circ}$, using six volts for illumination. Aqueous cells are counted in a column of light $0.5 \mathrm{~mm}$ wide by $2 \mathrm{~mm}$ high incident at $45^{\circ}$.

Disease of the posterior segment: Colour or red-free photographs and fluorescein angiograms are used for scoring pigment epithelial and choroidal changes, papillitis and optic atrophy. Scoring of posterior segment changes has been based on well recognised patterns of retinal and optic nerve disease in onchocerciasis. (Table IV) The system takes account of both the intensity and distribution of disease.

The initial retinal pigment epithelial changes appear characteristically as irregularity of the pigmentation $10^{\circ}$ temporal to the fovea, or occasionally nasal to the optic disc (Fig. 1). With advancing disease there is gradual extension of pigment epithelial changes around the macula, and intensification of atrophy. Increased intensity of atrophy and extension of disease do not necessarily go hand in hand so that each is considered separately (Fig. 2). The typical end stage appearance of the 'Ridley fundus' in which the whole macula is affected and there is reactive hyperpigmentation is awarded a score of 20 under both distribution and atrophy (Fig. 3).

Early papilitis is detected only by fluorescein angiography, (Fig. 4) but in the later stages disc hyperaemia and swelling become evident ophthalmoscopically. The swelling is usually segmental initially, but involves the whole disc with advancing disease. The earliest sign of loss of ganglion cell axons is identified as linear defects in the reflex of the inner retina. Segmental then overall disc pallor and total loss of nerve fibre bundles may occur in advanced optic nerve atrophy. These signs can be recognised on both colour and red-free photographs.

Table IV. Scoring system for disease of the posterior segment

\begin{tabular}{|c|c|c|c|c|}
\hline \multicolumn{3}{|l|}{ Feature } & \multicolumn{2}{|l|}{ Grades } \\
\hline $\begin{array}{l}\text { Pigment epithelial atrophy: } \\
\text { distribution: }\end{array}$ & temporal to macula & more than temporal & continuous round macula & whole of macula \\
\hline intensity: & RPE mottling & plus* atrophy & continuous* atrophy & $\begin{array}{l}\text { plus } \\
\text { hypertrophy }\end{array}$ \\
\hline Score & 5 & 10 & 15 & 20 \\
\hline $\begin{array}{l}\text { Optic neuritis: } \\
\text { Colour film: } \\
\text { hyperaemia: } \\
\text { swelling: }\end{array}$ & sectorial & overall & sectorial & overall \\
\hline $\begin{array}{l}\text { Angiographic leakage: } \\
\text { within OD: } \\
\text { beyond OD margin: }\end{array}$ & & sectorial & $\begin{array}{l}\text { overall } \\
\text { sectorial }\end{array}$ & overall \\
\hline Score & 5 & 10 & 15 & 20 \\
\hline $\begin{array}{l}\text { Optic Atrophy: } \\
\text { colour: } \\
\text { red free: }\end{array}$ & $\begin{array}{l}\text { linear nfb loss } \\
\text { linear nfb loss }\end{array}$ & $\begin{array}{l}\text { sectorial atrophy } \\
\text { sectorial nfb loss }\end{array}$ & $\begin{array}{l}\text { overall atrophy } \\
\text { total nfb loss }\end{array}$ & $\begin{array}{l}\text { atrophy with pigment } \\
\text { total nfb loss with pigment }\end{array}$ \\
\hline Score & 5 & 10 & 15 & 20 \\
\hline
\end{tabular}

Other Onchocercal**; vasculitis, CWS, Haemorrhage etc.: score 5 for each.

Other non-onchocercal: annotate but do not score.

Notes

If increase of any abnormality is identified but is insufficient to change the category, add 1 to the previous score.

*Plus atrophy-less than $50 \%$ of the affected area atrophic; continuous atrophy-50\% or more atrophic.

**Annotate the nature of change. Once an event has occurred the score will be reflected in subsequent visits whether or not the lesion still exists. Scores will accumulate as more events are recorded. 


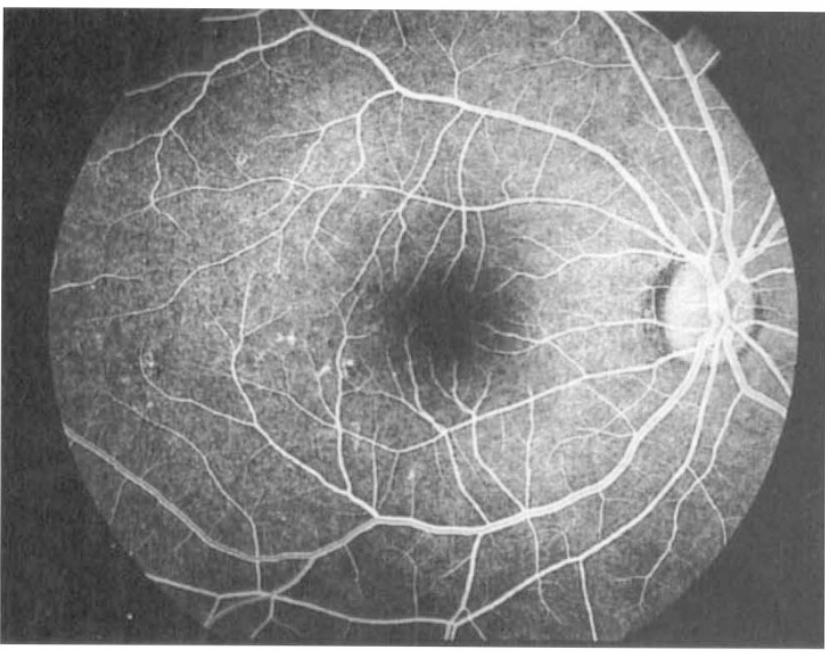

Fig 1. Fundus fluorescein angiogram of the right eye showing pigment epithelial defects at the temporal aspect of the macula.

Other changes attributable to onchocerciasis such as vasculitis, cotton wool spots and retinal haemorrhages each receive a score of five. The scores for these lesions are carried over to scores for subsequent visits even if the lesions disappear, as they represent destructive retinal processes.

Photographs are scored by two masked observers, any difference between the two scores being decided after consultation. The practicability of the system for posterior segment changes was tested by two masked observers scoring colour and red-free photographs, and fluorescein angiograms of 120 healthy males aged $18-50$ years who were recruited to chemotherapeutic trials. The concordance was between $85 \%$ and $90 \%$ for the different attributes of fundus disease.

Baseline scores and the effect of treatment: Changes occurring during therapy may be registered if there is a change in the category of any lesion. If there is progression of a lesion which is insufficient to change category, one is

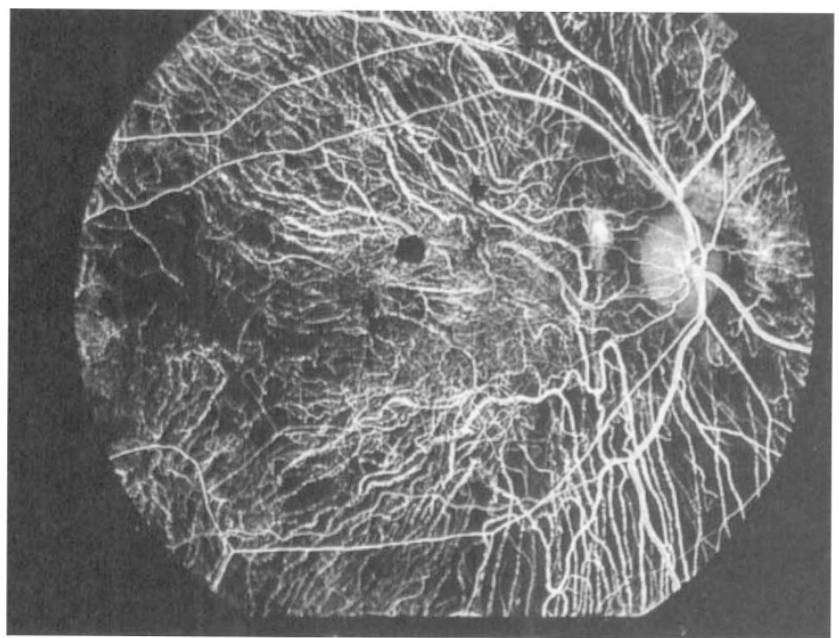

Fig 3. Fundus fluorescein angiogram of the right eye, venous phase, showing extensive atrophy of the retinal pigment epithelium.

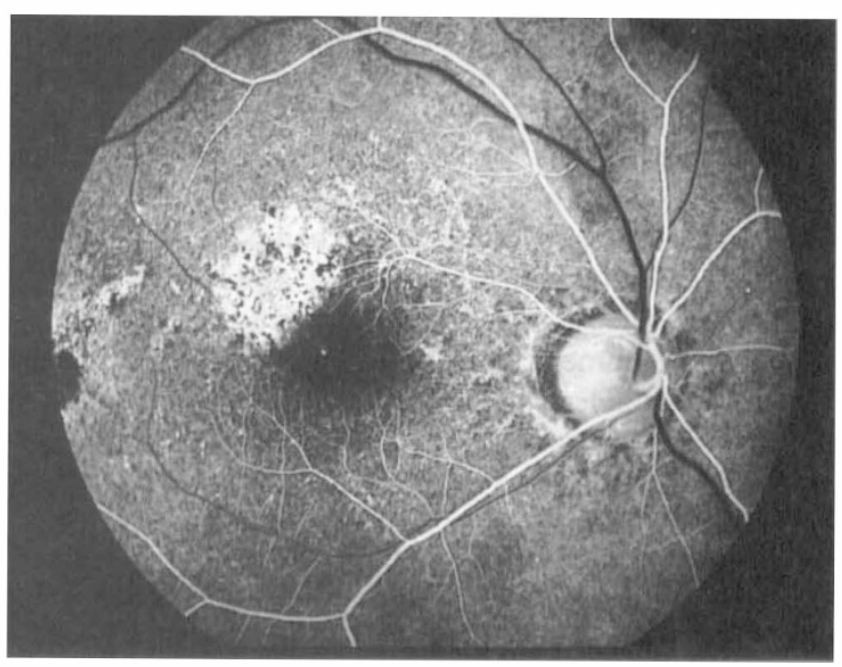

Fig 2. Fundus fluorescein angiogram of the right eye demonstrating atrophy of the pigment epithelium with scattered pigment epithelial hypertrophy masking choroidal fluorescence.

added to the previous score. Thus, four episodes of deterioration may be recorded without changing category.

Clinical information is entered into a computerised database which has been programmed to award appropriate scores for the clinical findings. Individual scores for each separate feature when added together produce the total score for that patient. This allows changes to be detected for each clinical attribute and for a patient as a whole over time. By recording change, it also enables comparison of ocular reactions between different treatment groups in a therapeutic trial, and the important reaction patterns to treatment may be identified by analysing individual components of disease separately.

\section{DISCUSSION}

The parameters chosen for this assessment have been selected to represent certain features found in untreated disease which may be aggravated by chemotherapy and adversely affect visual outcome. Symptoms have been

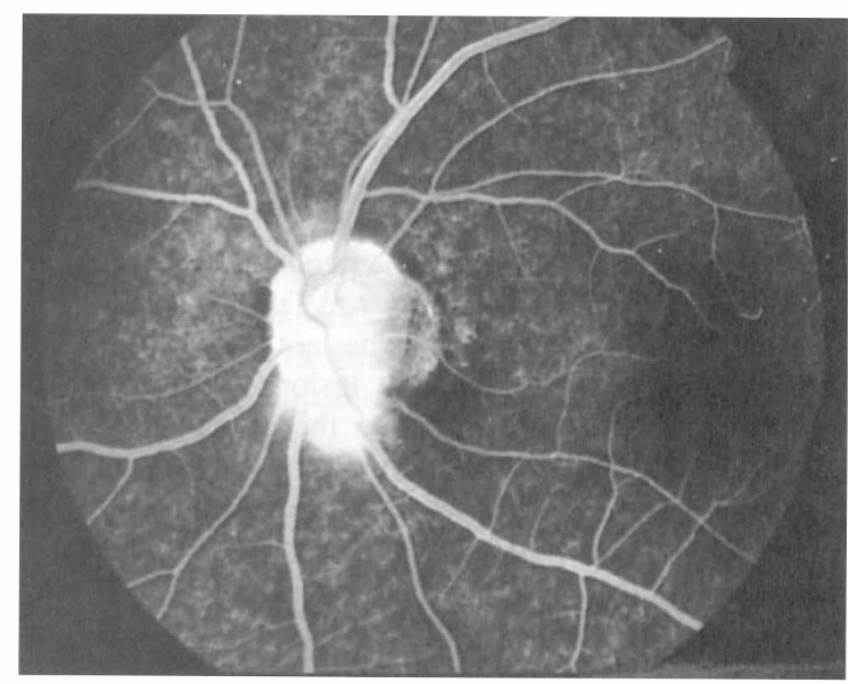

Fig 4. Late phase fundus fluorescein angiogram of the left eye. Note sectorial fluorescein leak from the disc extending into the retina. 
included in this system as they represent the patient's conception of his disease, and a drug causing severe symptoms may prove unacceptable for community use. However, owing to the difficulty in assessing ocular symptoms, the maximum score per patient has been limited to five to prevent undue weight being given to this feature. It is evident that the scoring system for specific features of disease cannot be considered as equivalents, and that the numerical progression in any aspect is not linear. Nevertheless the system includes those aspects of disease which are important indicators of progression, and some weighting can be given to those features which are most threatening to vision. Above all it allows change to be documented over time, and provides a protocol which can be used to detect reaction to treatment.

Unlike the 'reaction index' by Keyvan-Larijani et al ${ }^{10}$ microfilarial counts in the anterior chamber and cornea are not included in scoring disease. Ocular microfilarial counts following therapy are determined by both microfilarial migration and death. Ivermectin has been shown to increase anterior chamber parasite counts without inducing significant inflammation as part of the therapeutic effect such that it should not be considered an adverse reaction. $^{12}$ Incorporation of corneal microfilarial counts would lead to an increased score early in disease, thus giving the erroneous impression of a complication of therapy. Furthermore, a rise in the score attributable to ocular inflammatory changes could be masked in the total score by a subsequent fall in ocular microfilarial counts. This is analogous to the procedure employed in the quantification of systemic reactions where skin microfilarial counts are not utilised. . $^{7-9}$

It is well accepted that chorioretinal changes and optic atrophy account for significant visual morbidity in onchocerciasis, and aggravation of posterior segment disease represents the major threat from treatment. Typically good acuity is maintained until late in the disease when visual loss may be precipitate. Visual field loss however is an early manifestation of optic nerve involvement making it essential to detect and quantify visual field loss. Therapy with microfilaricidal drugs inducing an inflammatory reaction around microfilariae, such as diethylcarbamazine citrate (DEC-C), may accelerate optic nerve disease leading to permanent loss of visual function. ${ }^{6}$ Transient pigment epithelial lesions detectable only on fluorescein angiography have been demonstrated following DEC-C administration. In direct contrast to DEC-C, Ivermectin, also an effective microfilaricide has not yet been shown to induce fresh posterior segment lesions or to accelerate pre-existing disease. ${ }^{12}$ Owing to the unpredictable and potentially damaging effects of filaricidal drugs on the evolution of disease of the posterior segment any method for quantifying reactions to onchocerciasis therapy must include this feature.
Our aim in constructing the method described in this paper is to summarise numerically adverse ocular reactions to filaricide therapy in a way which complements the assessment of systemic reactions but with due regard being given to the cumulative effects of ocular damage. It also permits the comparison of adverse reactions and identification of particular reaction patterns to different chemotherapeutic regimens.

This investigation received financial support from the World Health Organisation Onchocerciasis Chemotherapy Project of the Onchocerciasis Control Programme in West Africa (Research Proposal No. 85006).

Key words: Key words: Chemotherapy, Eye, Microfilaricides, Onchocerciasis.

\section{REFERENCES}

1. Bryceson ADM, Warrell DA, Pope HM: Dangerous reactions to treatment of onchocerciasis with diethylcarbamazine. Br Med Journal 1977, 1: 742-44.

2. Awadzi K and Gilles HM: The chemotherapy of onchocerciasis III: A comparative study of diethylcarbamazine (DEC) and metrifonate. Ann Trop Med Parasitol 1980, 74: 199-210.

3. Greene BM, Taylor HR, Brown EJ et al.: Ocular and systemic complications of diethylcarbamazine therapy on onchocerciasis. Association with circulating immune complexes. J Infect Dis 1983, 147: 890-7.

4. Anderson $\mathrm{J}$ and Fuglsang $\mathrm{H}$ : Effects of diethylcarbamazine on ocular onchocerciasis. Tropenmed Parasitol 1976, 27: 263-78.

5. Bird AC, El-Sheikh H, Anderson J, Fuglsang H: Changes in visual function and in the posterior segment of the eye during treatment of onchocerciasis with diethylcarbamazine citrate. Br J Ophthalmol 1980, 64: 191-200.

6. Taylor HR, Greene BM: Ocular changes with oral and transepidermal diethylcarbamazine therapy of onchocerciasis Br J Ophthalmol 1981, 65: 494-502.

7. Awadzi K: The chemotherapy of onchocerciasis II. Quantitation of the clinical reaction to microfilaricides. Ann Trop Med Parasitol 1980, 74: 190-7.

8. Greene BM, Taylor HR, Cupp EW et al.: Comparison of ivermectin and diethylcarbamazine in the treatment of onchocerciasis. N Engl J Med 1985, 313: 133-8.

9. De Sole G, Remme J, Awadzi K et al.. Adverse reactions after large-scale treatment of onchocerciasis with ivermectin: combined results from eight community trials. Bull of the World Health Org 1989, 67: 707-19.

10. Keyvan Larijani E, Newland HS, Taylor HR: An ocular reaction index for use in the study of onchocerciasis. Tropenmed Parasitol 1985, 36: 241-3.

11. Bird AC, Anderson J, Fuglsang H: Morphology of posterior segment lesions of the eye in patients with onchocerciasis. BrJ Ophthalmol 1976, 60: 2-20.

12. Dadzie KY, Bird AC, Awadzi K, Schultz-Key H, Gilles HM, Aziz MA: Ocular findings in a double-blind study of ivermectin versus diethylcarbamazine versus placebo in the treatment of onchocerciasis. Br J Ophthalmol 1987, 71: 78-85. 